\title{
Is the golden hour optimally used in South Africa for children presenting with polytrauma?
}

\author{
J Zuidgeest, A Jonkheijm, M van Dijk, A van As \\ Department of Pediatric Surgery, Erasmus MC-Sophia Children's Hospital, Rotterdam, The Netherlands \\ J Zuidgeest, medical student \\ A Jonkheijm, medical student \\ $\mathrm{M}$ van Dijk, PhD \\ Department of Paediatric Surgery, Red Cross War Memorial Children's Hospital and University of Cape Town \\ A van As, MBA, FCS (SA), PhD
}

Corresponding author: A van As (sebastian.vanas@uct.ac.za)

Background. The major paediatric public health problem worldwide is injury or trauma. In 2004, 950000 children died as a result of injury. Objective. The aim of this study was to evaluate the logistics of medical care after paediatric polytrauma within the first hours after arrival into a trauma unit - the so-called Golden Hour.

Methods. Children presenting with polytrauma to the Trauma Unit at the Red Cross War Memorial Children's Hospital between May 2011 and August 2011 were considered for inclusion in the study.

Results. Fifty-five children were included in the final analysis. The median duration of stay in the Trauma Unit was 205 minutes (interquartile range 135 - 274).

Conclusion. Several factors were identified that unnecessarily prolonged the time that patients stayed in the trauma unit following arrival in hospital for polytrauma management.

S Afr Med J 2013;103(3):166-167. DOI:10.7196/SAMJ.6402

Worldwide, the major cause of morbidity, disability and mortality in children aged 1 - 18 years is polytrauma, which claimed the lives of 950000 children in 2004. ${ }^{[1]}$ Besides the physical effects of trauma, the mental health and well-being of children, and of their families, is adversely affected by such trauma. ${ }^{[2]}$ Effective treatment within the first hours - the golden hour - after admission to a hospital is critical to mitigating all these consequences. The term 'golden hour' (GH) was coined to describe the critical time period of a few minutes to several hours following traumatic injury. ${ }^{[3]}$ During this period, appropriate and effective medical treatment may prevent death. For children, the GH may be even more significant than for adults, since they are more vulnerable, owing to their smaller body size and more complex anatomy and physiology. ${ }^{[4]}$ A study performed in England and Wales revealed that $44 \%$ of children with trauma died within minutes, $20 \%$ within a few hours, and $36 \%$ within days. ${ }^{[4]}$

Children presenting with polytrauma (more than one injury) are frequently haemodynamically unstable at presentation. ${ }^{[5]}$ Imaging is crucial in the evaluation of children presenting after polytrauma because clinical examination tends to be unreliable ${ }^{[6]}$ It is estimated that $30 \%$ of children with severe trauma die because of inadequate evaluation on arrival in hospital. ${ }^{[5]}$ Accurate evaluation, early initiation of critical care measures and early radiographic imaging have the potential to prevent these deaths. ${ }^{[5,6]}$ For these reasons, efficient care from transport to hospital to admission to the paediatric intensive care unit (PCU) should be the goal.

This study evaluates the logistics of medical care of paediatric patients presenting with polytrauma within the first hours after arrival at the Trauma Unit of the Red Cross War Memorial Children's Hospital (RXCH) in Cape Town.

\section{Methods}

Study design

The study comprised a retrospective review of patients' medical records.

\section{Patients and setting}

Between May and August 2011, all paediatric patients aged 0 - 12 years who were referred to the Trauma Unit at the RXCH were eligible for inclusion. Approval of the Ethics Committee of the University of Cape Town was obtained.

\section{Procedure}

The admission book at the Trauma Unit was used to identify all patients presenting with polytrauma. All children who underwent full-body imaging (by LODOX scanner) and head or body computed tomography $(\mathrm{CT})$ or who were admitted to the Paediatric Intensive Care Unit (PICU) were included.

The patient's medical record was used to collect all data. Various time points were used for evaluation. These were T0 (time to hospital), T1 (time between arrival at Trauma Unit and LODOX scan), T2 (time between LODOX scan and CT scan), T3 (time between CT scan and admission) and T4 (time between arrival at the Trauma Unit and admission).

\section{Data analysis}

Descriptive statistics were used; normally distributed variables are presented as mean (standard deviation) and non-normally distributed variables as median (interquartile range (IQR)). Analyses were performed using SPSS 17.0. A $p$-value $<0.05$ (two-tailed) was considered statistically significant. The Kruskal-Wallis test served to compare waiting times from arrival in the Trauma Unit until 
admission to the PCU or trauma ward (T4) between groups with different types of injury and for differences between type of referral in respect of time to hospital following injury (T0) and time to admission either to the trauma ward or PCU following arrival in the Trauma Unit (T4).

\section{Results}

Fifty-five children with a median age of 6 years were included in the study. More than half had been referred by another clinic or hospital $(50.9 \%, n=28)$. Traffic-related injuries were the leading cause of trauma $(67.3 \%, n=37)$ followed by falls $(14.5 \%, n=8)$, burns $(14.5 \%$, $n=8)$ and others $(3.6 \%, n=2)$.

Information on the time interval between incident and arrival at the Trauma Unit (T0) was available for only 16 patients, and varied from 20 - 360 minutes, with a median of 82 minutes. Time to the hospital (T0) was significantly longer for children referred from another clinic or hospital ( $p=0.012$, Kruskal-Wallis test) compared with children who were brought directly to hospital without referral.

After arrival in the Trauma Unit, the waiting time for the total body imaging (T2) and the waiting time for the CT scan (T3) was documented for 45 patients. Total body imaging (T2) was completed after a median of 62 (IQR $44-85)$ minutes. The time from T2 to CT scan varied from 15 to 252 (median 40) minutes (Fig. 1).

One child died 410 minutes after arrival. Thirteen children were transferred to the PICU. One child had an operation of 55 minutes' duration before admission to the PCU. The median time interval between arrival at the hospital and transfer to the PCU (T4) in 10 patients was 240 (range 30 - 660) minutes (Fig. 1).

The waiting time (median 225 (IQR 211 - 303) minutes) from arrival at the Trauma Unit until admission (T4) varied with the type of injury and was significantly longer for children involved in a motor vehicle accident than those with other causes of trauma (burns, falls

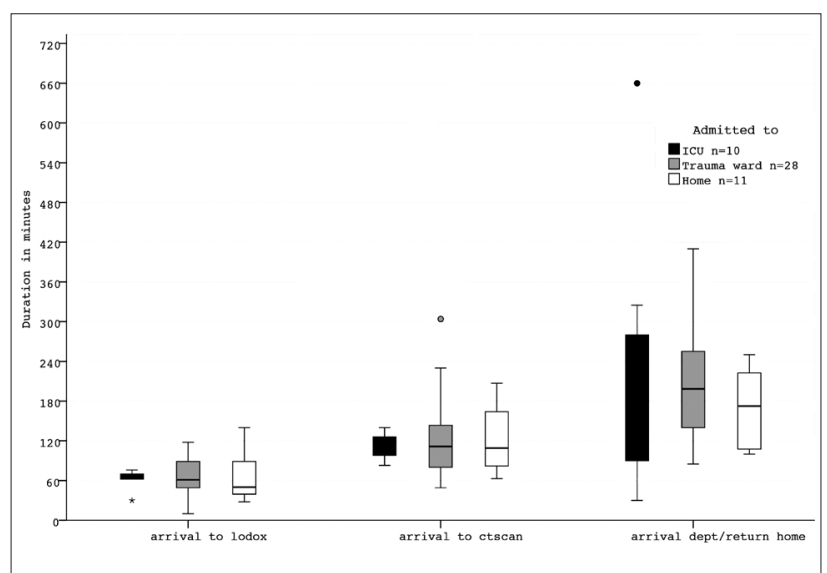

Fig. 1. Duration of stay at the Trauma Unit in minutes for 3 different types of admission $(\mathrm{n}=49)$. and others) ( $p=0.037$, Kruskal-Wallis test). Time between arrival at the Trauma Unit to admission was unrelated to whether referred or presenting directly to hospital ( $p=0.68$, Kruskal-Wallis test).

\section{Discussion}

The main finding of this study was the lengthy time spent in the Trauma Unit by patients before admission to either the trauma ward or PCU. The time interval (T2) between arrival at the Trauma Unit and total body imaging was 62 minutes, despite the fact that the scanner is situated in the resuscitation room. This period is significantly longer than the reported time interval of 6 minutes in adults. ${ }^{[7]}$ The immediate performing of total body imaging permits evaluation of the extent of injuries and provides direction for emergency management, and is of particular importance in children, since medical history may be unreliable, particularly in those who cannot yet talk, are sleepy, or are comatose and/or intubated. ${ }^{[6]}$

Reasons for delay in management following arrival in the Trauma Unit were numerous, including delay in registration and issuing a hospital folder, and lack of available and appropriately trained radiographic staff to undertake body imaging and scanning. (During after-hours and weekends, only 1 radiographer is on call for the entire hospital, frequently leading to delays in imaging.) In cases where a neurosurgeon and/or paediatric surgeon was consulted but was in theatre, there was additional delay. Other reasons for long waiting times between radiological imaging and admission to either the ICU or trauma ward were shift changes in nursing staff and unavailability of beds.

Patient factors also played a role, some children arriving unaccompanied by anyone who could provide a reliable history or consent for surgery.

\section{Conclusions}

In paediatric patients presenting with polytrauma, several factors account for delays in initiating hospital care. Changes can be made at the various bottlenecks identified in this study to mitigate the delay that potentially adversely affects morbidity and mortality, and so achieve optimal use of the golden hour.

\section{References}

1. Peden M. World report on child injury prevention appeals to "Keep Kids Safe". Inj Prev 2008;14(6):413-414. 2. Upperman JS, Burd R, Cox C, et al. Pediatric applied trauma research network: a call to action. J Trauma 2010;69(5):1304-1307.

Little WK. Golden hour or golden opportunity: Early management of pediatric trauma. Clin Ped Emerg Med 2010;11(1):4-9.

Samuels M, Wieteska S. Advanced Paediatric Life Support: The Practical Approach. 5th ed. Chichester John Wiley \& Sons, 2011:404.

5. Browne GJ, Cocks AJ, McCaskill ME. Current trends in the management of major paediatric trauma. Emerg Med 2001;13(4):418-425.

6. Willner EL, Jackson HA, Nager AL. Delayed diagnosis of injuries in pediatric trauma: the role of radiographic ordering practices. Am J Emerg Med 2012;30(1):115-123.

Beningfield S, Potgieter H, Nicol A, et al. Report on a new type of trauma full-body digital X-ray machine. Emerg Radiol 2003;10(1):23-29.

Accepted 30 October 2012 